\title{
Caracterización de la infección urinaria y resistencia antimicrobiana en receptores de trasplante renal de un centro colombiano
}

\section{Characterization of Urinary Tract Infection and Antimicrobial Resistance in Kidney Transplant Recipients from a Colombian Tertiary Center}

\author{
David Andrés Castañeda-Millán ${ }^{1,2(0)}$ Juan Carlos Osorio-Iriarte ${ }^{3}$ Juan Pablo Alzate-Granados²0 \\ Daniel Amórtegui-Rodríguez ${ }^{4}$ Juan Sebastián Arbeláez-Teuzaba ${ }^{5}{ }^{\circledR}$ María Camila Romero-Sánchez ${ }^{6}$ \\ Karen Flórez-Barbosa ${ }^{7}$ William Fajardo-Cediel ${ }^{7}$
}

1 Unidad de Urología, Facultad de Medicina. Universidad Nacional de Colombia. (Bogotá, Colombia)

${ }^{2}$ Grupo de Investigación e Innovación en Urología, Departamento de Cirugía. Facultad de Medicina. Universidad Nacional de Colombia. (Bogotá, Colombia)

3 Unidad de Urología, Universidad del Bosque. (Bogotá, Colombia)

${ }^{4}$ Facultad de Medicina, Fundación Universitaria Juan N. Corpas. (Bogotá, Colombia)

${ }^{5}$ Facultad de Medicina, Universidad de los Andes. (Bogotá, Colombia)

${ }^{6}$ Facultad de Medicina, Universidad Libre de Colombia. (Cali, Colombia)

${ }^{7}$ Grupo de Trasplante Renal, Fundación Clínica Shaio. (Bogotá, Colombia)

Urol Colomb 2021;30(3):e165-e170.

\author{
Address for correspondence Castañeda-Millán David Andrés, MD, \\ MSc, Unidad de Urología, Departamento de Cirugía. Facultad de \\ Medicina, Universidad Nacional de Colombia \\ (e-mail: dacastanedam@unal.edu.co).
}

\section{Resumen}

Palabras clave

- Infecciones urinarias

- fármacorresistencia microbiana

- trasplante de riñón
La infección del tracto urinario (ITU) es una de las principales complicaciones postrasplante renal, los datos a nivel nacional en ese grupo poblacional son limitados. Objetivos caracterizar la microbiología de las ITU presentadas en receptores de trasplante renal (TxR) en un centro colombiano durante el periodo 2017-2019, los factores relacionados con la resistencia antimicrobiana y el impacto de la ITU en la función del injerto renal.

Métodos estudio de corte transversal ejecutado mediante el análisis de la base de datos de ingresos hospitalarios por urgencias de pacientes receptores de TxR con sospecha clínica de ITU en una institución de cuarto nivel en Bogotá, Colombia. El análisis de datos se ejecutó en STATA 13.0.

Resultados La ITU causó $12,69 \%$ de visitas a urgencias en pacientes trasplantados. Los microorganismos aislados fueron: Escherichia coli 52,22\%, Klebsiella pneumoniae $16,67 \%$, Pseudomonas aeruginosa 4,44\%, Salmonella spp 4,44\%, Proteus mirabilis received

July 27,2020

accepted after revision

February 4, 2021
DOI https://doi.org/ $10.1055 / \mathrm{s}-0041-1730360$ ISSN 0120-789X. e ISSN 2027-0119.
(C) 2021. Sociedad Colombiana de Urología. All rights reserved. This is an open access article published by Thieme under the terms of the Creative Commons Attribution-NonDerivative-NonCommercial-License, permitting copying and reproduction so long as the original work is given appropriate credit. Contents may not be used for commercial purposes, or adapted, remixed, transformed or built upon. (https://creativecommons.org/ licenses/by-nc-nd/4.0/)

Thieme Revinter Publicações Ltda., Rua do Matoso 170, Rio de Janeiro, RJ, CEP 20270-135, Brazil 
$3,33 \%$, Serratia marcescens 2,22\%, Klebsiella oxytoca 2,22\%, Citrobacter koseri $1,11 \%$, Enterobacter cloacae 1,11\%, otros 2,22\%; El urocultivo fue negativo en $10 \%$ de los casos. El 28,39\% (n:23) de gérmenes aislados fue multisensible mientras que el 71,60\% (n:58) expresó algún tipo de patrón de resistencia distribuido así: 68,96\% productor de betalactamasa de espectro extendido (BLEE), 15,52\% productor de carbapenemasas, $12,06 \%$ productor de betalactamasa tipo IRT, $3,45 \%$ fue catalogado como multirresistente. $17,78 \%$ de los pacientes presentó criterios de urosepsis, no se registró ningún caso de mortalidad asociada a la ITU. La creatinina sérica tuvo un incremento promedio de $0,46 \mathrm{mg} / \mathrm{dl}$ durante el episodio de ITU $(p:<0,0001)$ y el antecedente de diabetes mellitus se relacionó con la ITU causada por gérmenes resistentes $(p: 0,008)$.

Conclusiones La ITU es una causa frecuente de atención en urgencias para pacientes receptores de TxR; la Escherichia coli es el microorganismo causal más frecuente y cerca del 70\% de los gérmenes aislados presentó algún patrón de resistencia antimicrobiana.

\begin{abstract}
Urinary tract infection (UTI) is one of the most common complications after kidney transplantation (KTx). This study aims to characterize the microbiology of UTIs presented in KTx recipients in a Colombian tertiary center during the period 2017-2019, factors related with antimicrobial resistance and the impact of UTI on kidney graft function.

Methods A cross-sectional retrospective single center study were made through the institutional database analysis of hospital admissions to the emergency room of KTx recipients with clinical suspicion of UTI. Data analysis was run on STATA 13.0.

Results UTI caused $12.69 \%$ of visits to the emergency room in transplant patients during the study period. The isolated microorganisms were Escherichia coli $52.22 \%$, Klebsiella pneumoniae $16.67 \%$, Pseudomonas aeruginosa $4.44 \%$, Salmonella spp $4.44 \%$, Proteus mirabilis 3.33\%, Serratia marcescens 2.22\%, Klebsiella oxytoca $2.22 \%$, Citrobacter koseroi $1.11 \%$, others $2.22 \%$; Urine culture was negative in $10 \%$ of cases. $28.39 \%$ ( $n: 23$ ) of isolated germs were multisensitive while $71.60 \%$ ( $n: 58)$ expressed some type of resistance pattern distributed as follows: $68.96 \%$ extended spectrum beta-lactamase (ESBL) producers, $15.52 \%$ carbapenemases producers, $12.06 \%$ IRT-type beta-lactamase

\section{Keywords}

- urinary tract infections

- drug resistance

- microbial

- kidney transplantation producers, $3.45 \%$ was classified as multi-resistant. $17.78 \%$ of patients presented criteria for urosepsis, there was no cases of mortality due to UTI. Serum creatinine had an average increase of $0.46 \mathrm{mg} / \mathrm{dl}$ during the UTI episode ( $\mathrm{p}:<0.0001)$ and a history of diabetes mellitus was related with UTI caused by resistant germs ( $p: 0.008$ ).

Conclusion UTI is a frequent cause of emergency care for $\mathrm{KTx}$ recipients. $E$. coli is the most common causative microorganism and about $70 \%$ of isolated germs showed some pattern of antimicrobial resistance.
\end{abstract}

\section{Introducción}

El trasplante renal (TxR) es la mejor opción de tratamiento para la enfermedad renal crónica terminal (ERCT), sin embargo, no es un procedimiento exento de riesgos o complicaciones. La naturaleza propia del procedimiento, la desfuncionalización del tracto urinario inferior que padecen los pacientes con ERCT y el uso de inmunomoduladores hacen que la infección del tracto urinario (ITU) sea una de las complicaciones postrasplante más frecuentes en pacientes receptores de TxR. ${ }^{1}$ Datos recientes evidencian un aumento progresivo del perfil de resistencia antimicrobiana en los patógenos aislados en pacientes receptores de TxR con ITU ${ }^{2}$ y también han abierto el debate sobre los posibles efectos de la ITU en la función del injerto renal. ${ }^{3}$ En Colombia existen pocos datos al respecto, por lo tanto, el presente estudio pretende caracterizar los episodios de ITU en la población receptora de TxR en una institución de cuarto nivel de Bogotá (Colombia), identificar factores relacionados con la severidad de la ITU y con la resistencia antimicrobiana en esa población, y esbozar los posibles efectos de los episodios de ITU en la función del injerto renal. 


\section{Métodos}

\section{Tipo de estudio}

Estudio de corte transversal.

\section{Población y muestra}

Se incluyeron todos los ingresos hospitalarios por urgencias de pacientes receptores de TxR con sospecha clínica de ITU en una institución de cuarto nivel en Bogotá (Colombia) durante el periodo enero/2017 a diciembre/2019.

\section{Variables}

Analizamos la cantidad de ingresos hospitalarios por urgencias en pacientes receptores de TXR con sospecha clínica de ITU (fiebre, escalofríos, síntomas urinarios irritativos) durante el periodo de estudio, el tipo de presentación clínica de la ITU, el tipo de microorganismo aislado y su patrón de resistencia antimicrobiana, el género y la edad de los pacientes, el tiempo desde el TxR, el tipo de esquema inmunosupresor, la función renal estimada mediante el valor absoluto de creatinina sérica y la mortalidad o perdida del injerto renal relacionada con el episodio de ITU en el grupo de estudio.

\section{Análisis estadístico}

Se ejecutó la prueba de $\mathrm{Chi}^{2}$ Pearson para evaluar diferencias en las variables cualitativas y el test T student para evaluar las diferencias en variables cuantitativas. Los resultados se expresan en medidas de frecuencia absoluta y relativa para las variables cualitativas y en forma de medidas de resumen y dispersión para las variables cuantitativas. El análisis de datos se ejecutó en STATA 13.0.

\section{Consideraciones éticas}

Según la legislación colombiana (Resolución 8430 de 1993) es un estudio sin riesgo, no requiere consentimiento informado. Se respetaron los principios establecidos en la declaración de Helsinki. Proyecto aprobado por el comité multidisciplinario de trasplante renal de la institución.

\section{Resultados}

La ITU causó $12,69 \%$ de visitas a urgencias en pacientes trasplantados durante el periodo de estudio (90 de 772 atenciones). Se registraron 90 episodios de ITU en 69 pacientes. De ellos, 66,67\% fueron del género femenino, y $33,33 \%$ del género masculino. La edad promedio de los pacientes fue de 50,01 años (19-76 años). El nivel promedio de creatinina basal en el grupo de estudio fue de $1,46 \mathrm{mg} / \mathrm{dl}$ $(0,6-4,0 \mathrm{mg} / \mathrm{dl})$, mientras que el nivel de creatinina sérico promedio durante el episodio de ITU fue de $1,93 \mathrm{mg} / \mathrm{dl}(0,5$ $-5,7 \mathrm{mg} / \mathrm{dl}$ ). El 20\% de los pacientes eran diabéticos. El 26,67\% de los pacientes se encontraban en régimen inmunosupresor estándar (tacrolimus + micofenolato + corticoide), 44,44\% en régimen inmunosupresor dual sin corticoide (tacrolimus + micofenolato), 2,22\% con régimen a base de inhibidores de la mTOR (mTOR + tacrolimus), 26,67\% se encontraba en otro tipo de régimen inmunosupresor
Tabla 1 Caracterización de los gérmenes causantes de ITU en receptores de TxR (2017-2019)

\begin{tabular}{|l|l|}
\hline Microorganismo & \% (n) \\
\hline Escherichia coli & $52,22(47)$ \\
\hline Klebsiella pneumoniae & $16,67(15)$ \\
\hline Pseudomonas aeruginosa & $4,44(4)$ \\
\hline Salmonella spp & $4,44(4)$ \\
\hline Proteus mirabilis & $3,33(3)$ \\
\hline Serratia marcescens & $2,22(2)$ \\
\hline Klebsiella oxytoca & $2,22(2)$ \\
\hline Citrobacter koseri & $1,11(1)$ \\
\hline Enterobacter cloacae & $1,11(1)$ \\
\hline Otros & $2,22(2)$ \\
\hline
\end{tabular}

Abreviaturas: ITU, Infección del tracto urinario; TxR, Trasplante renal.

(micofenolato + corticoide, ciclosporina + corticoide, un solo fármaco). El 53,34\% del total de pacientes incluían en su esquema inmunosupresor la presencia de corticoides. El 80\% de los pacientes recibieron injerto renal de donante cadavérico. El tiempo transcurrido desde el TxR hasta el episodio de ITU fue de menos de 1 año en el 12,22\%, entre 2 a 5 años en 28,89\%, 6 a 10 años en $26,67 \%$ y más de 10 años en $32,22 \%$ de pacientes.

Los microorganismos reportados en los urocultivos se describen en la - Tabla 1.

El urocultivo final fue negativo en el $10 \%$ de los casos. El 28,39\% (n:23) de gérmenes aislados fue multisensible mientras que un $71,60 \%$ (n:58) expresó algún tipo de patrón de resistencia distribuido así: 68,96\% productor de betalactamasa de espectro extendido (BLEE), 15,52\% productor de carbapenemasas, $12,06 \%$ productor de betalactamasa tipo IRT (betalactamasa tipo resistente a los inhibidores), 3,45\% fue catalogado como panrresistente (Ver -Figura 1). El $70 \%$ de los cuadros clínicos se presentaron inicialmente como ITU baja y $30 \%$ como ITU alta; $17,78 \%$ de los pacientes presentó criterios de sepsis de origen urinario, en $15,56 \%$ se detectó bacteriemia, sólo 5,56\% de pacientes requirió manejo en unidad de cuidados intensivos y no se registró ningún caso de mortalidad o pérdida del injerto renal asociada a la ITU.

Durante el episodio de ITU el $22,22 \%$ de pacientes no presentó cambios en su creatinina sérica basal mientras que un $77,77 \%$ presentó un incremento en el nivel basal de creatinina sérica; el cambio estuvo distribuido así: 50\% tuvo un incremento menor al $0,5 \mathrm{mg} / \mathrm{dl}, 13,33 \%$ entre 0,6 $1,0 \mathrm{mg} / \mathrm{dl}, 10 \%$ entre $1,1-2,0 \mathrm{mg} / \mathrm{dl}$ y $4,44 \%$ un incremento mayor a $2,1 \mathrm{mg} / \mathrm{dl}$ desde el nivel de creatinina basal (ver - Figura 2). Se evidenció una diferencia promedio de $0,46 \mathrm{mg} / \mathrm{dl}$ entre la creatinina basal y la creatinina durante el episodio de ITU (p-valor t-student $<0,0001$ ).

No se encontraron relaciones significativas entre el tipo de ITU (alta o baja) o la presencia de bacteriemia y el género ( $p$ : $0,62 ; p: 0,68)$, el antecedente de diabetes mellitus $(p: 0,42, p$ : $0,88)$, el tiempo desde el trasplante ( $p: 0,37, p: 0,48)$, el uso de corticoide $(p: 0,85)$, el esquema inmunosupresor $(p: 0,75)$. 


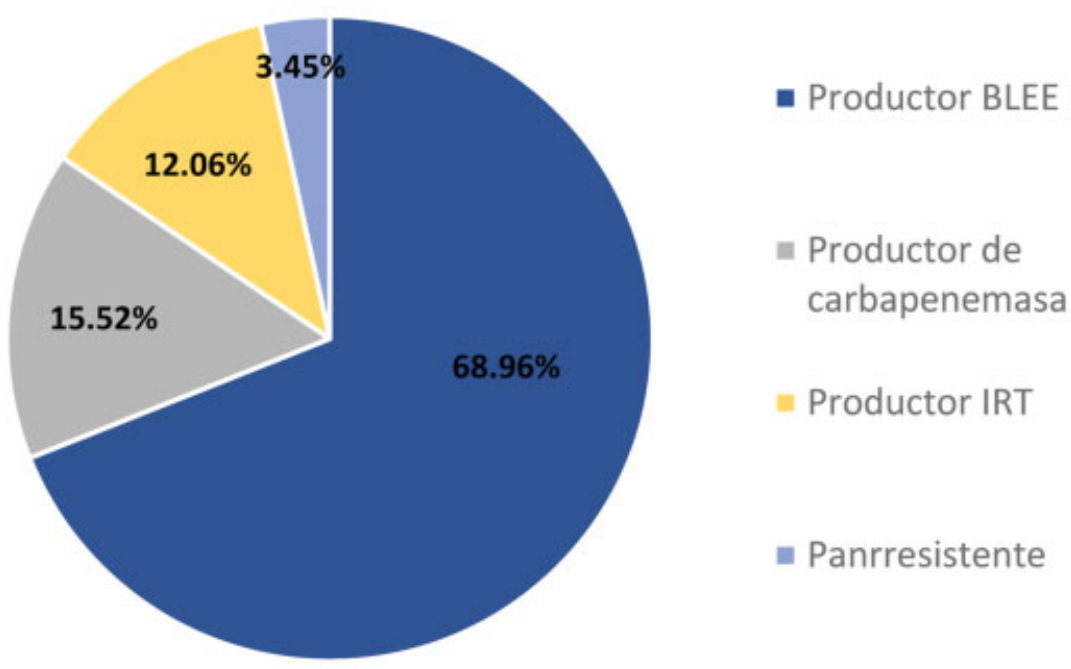

Fig. 1 Caracterización de la resistencia antimicrobiana de los gérmenes causantes de ITU en pacientes receptores de TxR (Correspondiente al $71,60 \%$ de aislamientos microbiológicos). BLEE: betalactamasa de espectro extendido, IRT: betalactamasa tipo resistente a los inhibidores, ITU: infección del tracto urinario, TxR: trasplante renal.

Sin embargo, se encontró una relación entre el tipo de esquema inmunosupresor (Tacrolimus + micofenolato y mTOR-Tacrolimus) y el tipo de ITU - alta $(p: 0,006)$ y en la presencia de resistencia bacteriana en pacientes con antecedente de diabetes mellitus ( $p$ : 0,008).

\section{Discusión}

La ITU es uno de los principales motivos de consulta y de requerimiento de manejo intrahospitalario en pacientes receptores de TxR, se estima que aproximadamente un 30\% de las hospitalizaciones por sepsis en pacientes receptores de TxR están vinculadas a la ITU, una incidencia de $4,5 \operatorname{casos}^{*} 100$ trasplantes/día ${ }^{4}$ y una prevalencia que en algunas series puede alcanzar hasta el $80 \%$ de pacientes en ese grupo poblacional. ${ }^{5}$

Se han descrito varios factores de riesgo para la presencia de ITU en pacientes receptores de TxR, dentro de ellos es preciso resaltar: género femenino del receptor, antecedente de diabetes mellitus en el receptor, periodo prolongado de diálisis, baja capacidad vesical pretrasplante, tipo de reconstrucción urinaria, utilización de catéter ureteral JJ, uso de injertos de donante cadavérico, balance neto de inmunosupresión, episodios de rechazo agudo del injerto, episodios de función retardada del injerto, infección por citomegalovirus en el receptor, trasplante renal dual, etiología urológica de ERCT entre otros. ${ }^{6-8}$

Los gérmenes gram negativos son los principales agentes etiológicos de ITU en receptores de TxR, los datos encontrados en ese grupo de estudio (ver - Tabla 1) son similares a los descritos en la literatura. ${ }^{9}$ Durante los últimos años, el aumento de la resistencia antimicrobiana ha generado serios debates a nivel mundial; la población receptora de TxR se encuentra sometida a un fenómeno de presión de selección para cepas de microorganismos resistentes como posible consecuencia del uso de estrategias de profilaxis antimicrobiana postrasplante, recientemente se determinó que cerca del 10\% (IC 95\%: 4-17\%) de las ITU en receptores de TxR son producidas por gérmenes gram negativos productores de BLEE. ${ }^{10}$ A diferencia de los datos mundiales, nuestro estudio evidenció un porcentaje mayor de gérmenes BLEE como agentes etiológicos de ITU en receptores de TxR, sin embargo, en términos generales de resistencia antimicrobiana en ITU se asemeja al $70 \%$ descrito por Cofán y cols. ${ }^{11}$

Los datos disponibles en Latinoamérica demuestran que el nivel de multirresistencia en ITU en pacientes con TxR en Argentina es del 31,18\% ${ }^{12}$; en Perú, 39\% de cepas de E. coli y $65 \%$ de K. pneumoniae productoras de ITU en receptores de TxR fueron BLEE y se documentó multirresistencia antimicrobiana en $37 \%$ de los casos. $^{13}$ Otro estudio colombiano $^{14}$ demostró multirresistencia en $41,5 \%$ de casos de infección bacteriana postrasplante y presencia de BLEE en $54,5 \%$ y $40,9 \%$ de cepas de E. Coli y K. pneumoniae productoras de ITU. En Brasil se evidenciaron gérmenes BLEE hasta en 57,5\% de episodios de ITU en receptores de TxR. ${ }^{15}$

Delmas y col., encontraron en su estudio ${ }^{16}$ un odss ratio de 2,29 [IC 95\%: 1,11-4,74, $p$ : $<0,05]$ para resistencia antimicrobiana a quinolonas o presencia de gérmenes BLEE en receptores de TxR con diabetes mellitus; de forma similar, ese estudio evidencia una relación significativa entre el antecedente de diabetes mellitus y la presencia de episodios de ITU por germen con resistencia antimicrobiana en receptores de TxR ( $p$ : 0,008).

Los resultados también ponen de manifiesto una posible relación entre el tipo de esquema inmunosupresor (tacrolimus + micofenolato y mTOR + tacrolimus) y el tipo de ITU (alta) que presentaron los pacientes $(p: 0,006)$, sin embargo, datos recientes ${ }^{17}$ no han demostrado diferencias significativas en el riesgo de ITU según el esquema inmunosupresor utilizado (anticalcineurínicos vs inhibidores de la mTOR) [OR: 0,86, IC 95\%: 0,71-1,75, p: 0,33].

Se demostró una diferencia significativa entre la creatinina basal de los receptores de TxR y la creatinina 


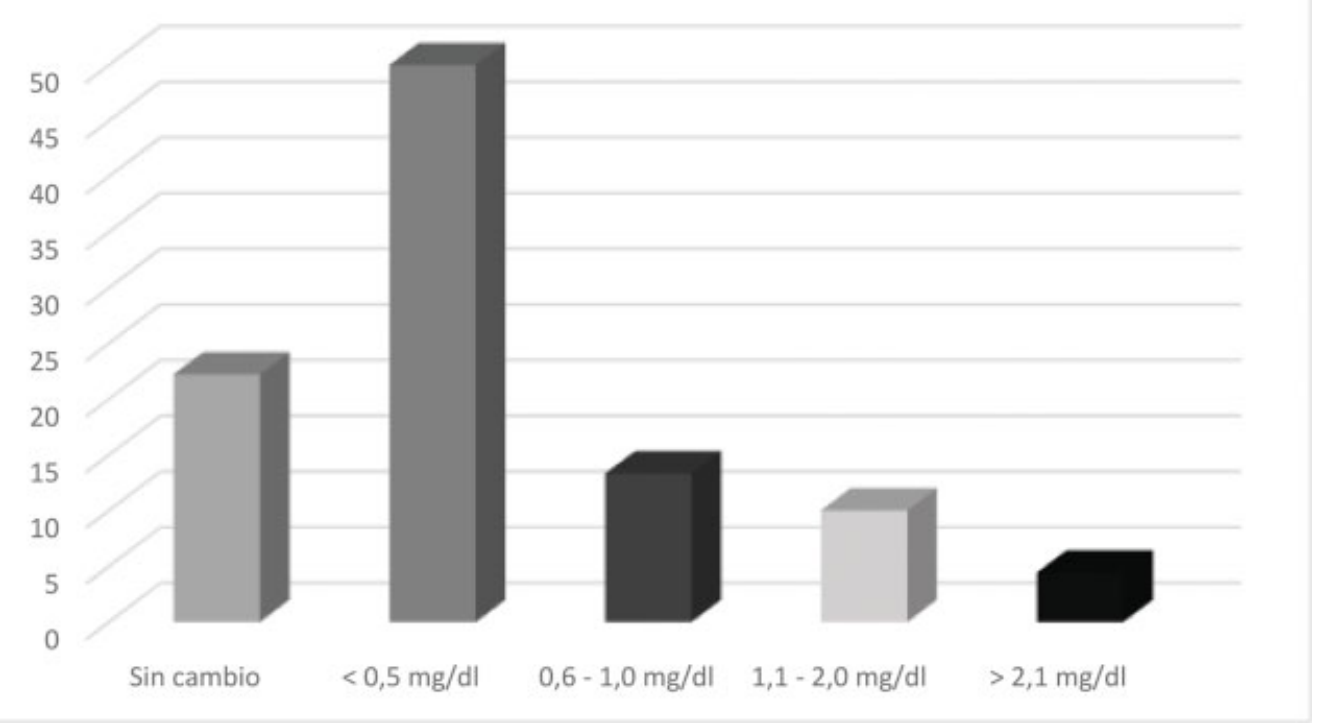

Fig. 2 Cambio de la creatinina sérica desde el nivel basal durante el episodio de infección urinaria en pacientes receptores de trasplante renal.

sérica durante el episodio de ITU $(p:<0,0001)$ con una posible significancia clínica acorde a las guías KDIGO, ${ }^{18} \sin$ embargo, los datos internacionales disponibles al respecto no son uniformes. Brodo y cols., ${ }^{19}$ han postulado a los episodios de ITU alta como un factor de riesgo para la alteración funcional del injerto renal en receptores de TxR [OR: 2,0. IC 95\%: 1,3-3,2, p: 0,001]; Ariza-Heredia y cols., ${ }^{20}$ no encontraron diferencia significativa en la tasa de filtración glomerular estimada ni en el valor absoluto de creatinina sérica en receptores de TxR con ITU ( $p$ : 0,646), sin embargo, en la estimación de la tasa de filtración glomerular con iotalamato encontraron una reducción de 5,09\% en pacientes receptores de TxR que sufrieron al menos un episodio de ITU ( $p$ : 0,044); Sing y cols., ${ }^{21}$ no encontraron diferencia en la función renal de receptores de TxR con ITU $(p: 0,38)$ ni en la proteinuria $(p: 0,14)$ tras un mes de ocurrido el evento infeccioso; Pesce y $\operatorname{cols}^{3}$ recientemente describieron la ITU recurrente como factor predictor independiente de la función del injerto renal [HR: 2,2. IC 95\%: 1,3-3,5, p: 0,001] y por último, Pinheiro y cols $^{22}$ definieron a la ITU causada por gérmenes productores de carbapenemasas como factor asociado a aumento de la mortalidad del receptor de TxR (HR: 2,62, IC 95\%: $1,37-5,00, p: 0,04)$ y a la disminución de la supervivencia del injerto renal (p:0,006).

A pesar de las limitaciones del presente estudio (derivadas del tamaño de muestra, método de selección, tipo de estudio, método de recolección y procesamiento de datos, entre otras), los resultados obtenidos aportan a la construcción epidemiológica de la ITU en receptores de TxR en Colombia y Latinoamérica, además permiten reconocer a la resistencia antimicrobiana como un problema cada vez más frecuente en la población receptora de TxR en la región, a la diabetes mellitus como un factor relacionado con la resistencia antimicrobiana en receptores de TxR y a la ITU como un factor que puede afectar negativamente la función del injerto renal y los desenlaces clínicos de los pacientes receptores de TxR.

\section{Conclusiones}

La ITU es una causa frecuente de atención en urgencias para pacientes receptores de TxR; la Escherichia coli es el microorganismo causal más frecuente y cerca del $70 \%$ de gérmenes aislados en el estudio presentó algún patrón de resistencia antimicrobiana, siendo el más frecuente la presencia de gérmenes BLEE. En el receptor de TxR, la diabetes mellitus es un factor que se relaciona con la resistencia antimicrobiana durante los episodios de ITU y se encontraron diferencias significativas entre la creatinina sérica durante el episodio de ITU comparada con su nivel basal.

Conflictos de interés

Los autores no tienen conflicto de intereses para declarar.

\section{Referencias}

1 Chacón-Mora N, Pachón Díaz J, Cordero Matía E. Urinary tract infection in kidney transplant recipients. Enferm Infecc Microbiol Clin 2017;35(04):255-259. Doi: 10.1016/j.eimc.2016.03.003

2 Korth J, Kukalla J, Rath PM, et al. Increased resistance of gramnegative urinary pathogens after kidney transplantation. BMC Nephrol 2017;18(01):164. Doi: 10.1186/s12882-017-0580-z

3 Pesce F, Martino M, Fiorentino M, et al. Recurrent urinary tract infections in kidney transplant recipients during the first-year influence long-term graft function: a single-center retrospective cohort study. J Nephrol 2019;32(04):661-668. Doi: 10.1007/ s40620-019-00591-5

4 Goldman JD, Julian K. Urinary tract infections in solid organ transplant recipients: Guidelines from the American Society of Transplantation Infectious Diseases Community of Practice. Clin Transplant 2019;33(09):e13507. Doi: 10.1111/ctr.13507

5 Hollyer I, Ison MG. The challenge of urinary tract infections in renal transplant recipients. Transpl Infect Dis 2018;20(02): e12828. Doi: 10.1111/tid.12828

6 Castañeda DA, León K, Martín R, López L, Pérez H, Lozano E. Urinary tract infection and kidney transplantation: a review of diagnosis, causes, and current clinical approach. Transplant Proc 
2013;45(04):1590-1592. Doi: 10.1016/j.transproceed.2013.01. 014

7 Fajardo-Cediel W, Flóres-Barbosa K. García-Cuevas J y cols. Capacidad vesical como pronóstico de trasplante renal. Urol Colomb 2020;29:32-38. Doi: 10.1055/s-0039-1696698

8 Fiorentino M, Pesce F, Schena A, Simone S, Castellano G, Gesualdo L. Updates on urinary tract infections in kidney transplantation. J Nephrol 2019;32(05):751-761. Doi: 10.1007/s40620-01900585-3

9 Ness D, Olsburgh J. UTI in kidney transplant. World J Urol 2020;38 (01):81-88. Doi: 10.1007/s00345-019-02742-6

10 Alevizakos M, Nasioudis D, Mylonakis E. Urinary tract infections caused by ESBL-producing Enterobacteriaceae in renal transplant recipients: A systematic review and meta-analysis. Transpl Infect Dis 2017;19(06):e12759. Doi: 10.1111/tid.12759

11 Cofán Pujol F, Cruzado Vega L. Errasti P y cols. Infecciones multirresistentes en el trasplante renal. Nefrologia Sup Ext 2018;9(02):67-81

12 Sciavelli R, Ajzenszlos M. Di Tullio D y cols. Infecciones urinarias por microorganismos multirresistentes en pacientes trasplantados renales internados. Rev Nefrol Dial Transpl 2019; 39(01):15-25

13 Méndez Chacón P, Bardales Viguria FArdiles Aniceto A y cols. Infección del tracto urinario en receptores de trasplante renal. An Fac med 2017;78(01):11-16. Doi: 10.15381/anales.v78i1.13015

14 Patiño-López M, Echeverri-Toro L. Bonfante-Olivares L y cols. Infecciones tempranas en pacientes trasplantados en un hospital de alta complejidad. Infectio 2017;21(03):148-153. Doi: 10.22354 /in.v21i3.671

15 Pinheiro HS, Mituiassu AM, Carminatti M, Braga AM, Bastos MG. Urinary tract infection caused by extended-spectrum beta- lactamase-producing bacteria in kidney transplant patients. Transplant Proc 2010;42(02):486-487. Doi: 10.1016/j. transproceed.2010.02.002

16 Delmas-Frenette C, Dorais M, Tavares-Brum A, et al. Epidemiology and outcome of antimicrobial resistance to gram-negative pathogens in bacteriuric kidney transplant recipients. Transpl Infect Dis 2017;19(04):e12722. Doi: 10.1111/tid.12722

17 Wolf S, Lauseker M, Schiergens T, et al. Infections after kidney transplantation: A comparison of mTOR-Is and CNIs as basic immunosuppressants. A systematic review and meta-analysis. Transpl Infect Dis 2020;22(03):e13267. Doi: 10.1111/tid.13267

18 KDIGO - Acute Kidney Injury Work Group. Kidney Disease: Improving Global Outcomes (KDIGO) clinical practice guideline for acute kidney injury. Kidney Int Suppl 2012;2:1-141. Doi: 10.1038/kisup.2012.7

19 Bodro M, Sanclemente G, Lipperheide I, et al. Impact of urinary tract infections on short-term kidney graft outcome. Clin Microbiol Infect 2015;21(12):1104.e1-1104.e8. Doi: 10.1016/j. cmi.2015.07.019

20 Ariza-Heredia EJ, Beam EN, Lesnick TG, Cosio FG, Kremers WK, Razonable RR. Impact of urinary tract infection on allograft function after kidney transplantation. Clin Transplant 2014;28 (06):683-690. Doi: 10.1111/ctr.12366

21 Singh R, Geerlings SE, Peters-Sengers $H$, et al. Incidence, risk factors, and the impact of allograft pyelonephritis on renal allograft function. Transpl Infect Dis 2016;18(05):647-660. Doi: 10.1111/tid. 12568

22 Freire MP, Mendes CV, Piovesan AC, et al. Does the urinary tract infection caused by carbapenem-resistant Gram-negative bacilli impact the outcome of kidney transplant recipients? Transpl Infect Dis 2018;20(04):e12923. Doi: 10.1111/tid.12923 